\title{
Estudio de la Amenorrea Primaria Durante el Período de 4 Años 1976 - 1980
}

\author{
Centro de Investigaciones Médicas \\ Universidad de Antioquia \\ Hospital Universitario San Vicente de Paúl
}

\author{
Carlos Javier Pilonieta P.* \\ Jairo García G. * * \\ José Luis Ramírez C.***
}

\section{INTRODUCCION}

Se considera como amenorrea primaria la ausencia de menstruación después de los 18 años de edad; no es muy frecuente, pero existen dificultades para llegar a su causa etiológica y para tomar una conducta. La amenorrea es sólo la manifestación clínica de una entidad que es.e necesario diagnosticar con el fin de dar un pronóstico e iniciar el tratamiento, logrando así tranquilizar a la paciente $y$ familiares que la rodean.

Universalmente se tiene el criterio de estudiar la paciente con amenorrea primaria después de los 18 años de edad, criterio que no compartimos si se tiene en cuenta que se pueden detectar tras-

\footnotetext{
* Residente IV Visitante Hospital Militar.

Médico Genetista, Departamento Morfología. U. de A.

**** Médico Gineco Endocrinólogo. Unidad de Ginecología Endocrina y Reproducción Humana, $U$. de $A$.
}

\author{
Guillermo Henao C. ${ }^{* * * *}$ \\ Jorge Mario Mejía R.**** \\ Alberto Abad C. \\ Fabio Sánchez $\mathrm{E}$. $^{* * * *}$
}

tornos en el desarrollo de la pubertad desde una época más precoz, basados en un análisis clínico programado, complementado con exámenes de laboratorio específicos $(1-2-3)$. Con base en esta aseveración se presentan los resultados obtenidos en un estudio de pacientes con amenorrea primaria efectuado durante un período de 4 años.

\section{Material y Métodos}

Se incluyen en el estudio las pacientes que consultaron por amenorrea primaria durante el período comprendido entre enero 1 de 1976 a diciembre 13 de 1979, a la consulta de Ginecología Endocrina y Reproducción Humana, del Departamento de Obstetricia y Ginecología,

**** Médico Endocrinólogo. Laboratorio de Endocrinología Clínica Soma.

\section{Reimpresos:}

Doctor Fabio Sánchez Escobar. Departamento de Obstetricia y Ginecología. Hospital San Vicente de Paúl. 
Universidad de Antioquia, Hospital Universitario San Vicente de Paúl, Medellín.

Todas las pacientes fueron referidas por los servicios de la consulta externa y los Centros de Salud del Municipio. De acuerdo a los hallazgos físicos se practicaron a las pacientes exámenes complementarios a saber: citología funcional, biopsia de endometrio, cromatina sexual, cariotipo y determinaciones hormonales: hormona folículo estimulante (FSH), hormona lutenizante (LH) y prolactina (PRL) en plasma; 17 cetosteroides y 17 hidroxiesteroides en orina de 24 horas.

Esta metodología difiere de otras ya publicadas $(4-5-6-7)$. Las pacientes estudiadas se clasificaron en 3 grupos, de acuerdo a su causa etiológica así:

\section{Anatómica:}

Si la causa de la amenorrea era a nivel del efector o de las vías de drenaje.

\section{Genética:}

Si la causa era el resultado de un trastorno cromosómico, de receptores o enzimático.

\section{Endocrina:}

Si la causa se debía a un trastorno funcional u orgánico en el eje hipotálamo, hipófisis u ovario.

\section{RESULTADOS}

De un total de 1.250 pacientes que consultaron a la unidad de Ginecología Endocrina y Reproducción Humana durante los 4 años del estudio, se diagnósticó amenorrea primaria en 53 pacientes, lo cual equivale a un promedio de 4 pacientes por cada $100(4 \%)$.

De las 53 pacientes, en $44(88.3 \%)$ se completó el estudio y llenaron los requisitos necesarios para un diagnóstico adecuado.
Del total de pacientes con amenorrea primaria, sólo el $22.6 \%$ tenían vida* sexual activa (tabla No. 1).

$$
\text { Tabla No. } 1
$$

CLASIFICACION DE 53 PACIENTES
CON AMENORREA PRIMARIA DE
ACUERDO AL ESTADO CIVIL
(1976-1979) H.U.S.V. DE P. - U. DE A.

\begin{tabular}{|c|c|c|}
\hline Estado Civil & No. de Pacientes & Porcentaje \\
\hline Casadas & 12 & $22.6 \%$ \\
\hline Solteras & 41 & 77.4 \\
\hline
\end{tabular}

El $60.4 \%$ de las pacientes consultaron tardíamente, después de los 20 años de edad y correspondió el mayor porcentaje al grupo entre los 20 a 24 años $(37.7 \%)$. Sólo el $5.7 \%$ lo hizo después de los 30 años (Tabla No. 2).

Tabla No. 2

DISTRIBUCION POR EDADES DE 53 PACIENTES CON AMENORREA PRIMARIA: H.U.S.V. DE P. - U. DE A., 1976-1979

\begin{tabular}{lcc}
\hline Años & No. de pacientes & Porcentaje \\
\hline 20 & 21 & 39.6 \\
$20-24$ & 20 & 37.7 \\
$25-29$ & 9 & 17.0 \\
30 ó más & 3 & 5.7 \\
\hline
\end{tabular}

El $79.2 \%$ de las pacientes presentaban caracteres sexuales secundarios en el momento de la consulta, de éstas el $22.6 \%$ habían recibido tratamiento hormonal sin diagnóstico clínico previo. Sólo 11 pacientes $(20.8 \%)$ ten ían infantilismo sexual, fenómeno que induce a una consulta más precoz (Tabla No. 3). 
Tabla No. 3

\section{CLASIFICACION DE 53 PACIENTES CON AMENORREA PRIMARIA DE ACUERDO A LA PRESENCIA DE CARACTERES SEXUALES SECUNDARIOS. H.U.S.V. DE P. - U. DE A. 1976-1979}

\begin{tabular}{|lcccc|}
\hline \multicolumn{3}{|c|}{ CARACTERES } & SEXUALES \\
\hline \multicolumn{3}{|c|}{ Infantiles } & \multicolumn{3}{c|}{ Presentes } \\
\hline & \multicolumn{3}{c|}{$\begin{array}{c}\text { Espontá- } \\
\text { neos }\end{array}$} & Inducidos \\
\hline Pacientes & 11 & 30 & 12 & 53 \\
Porcentaje & 20.8 & 56.6 & 22.6 & 100 \\
\hline \hline
\end{tabular}

En la Tabla No. 4 se aprecia que el $59.5 \%$ de las pacientes con desarrollo de la glándula mamaria consultaron después de los 20 años de edad, mientras que el $54.5 \%$ que presentaban ausencia de la glándula mamaria lo hicieron más precozmente.

Tabla No. 4

CLASIFICACION DE 53 PACIENTES CON AMENORREA PRIMARIA DE ACUERDO AL DESARROLLO DE LA GLANDULA MAMARIA H.U.S.V. DE P. - U. DE A., 1976-1979

GLANDULA MAMARIA

\begin{tabular}{ccc}
\hline Años & Presente & Ausente \\
\hline-20 & $17(40.5 \%)$ & $6(54.5 \%$ \\
+20 & $25(59.5 \%)$ & $5(45.5 \%)$ \\
Total & $42(100.0 \%)$ & $11(100.0 \%)$ \\
\hline \hline
\end{tabular}

El estudio de los factores etiológicos en las 44 pacientes con amenorrea se observa en la Tabla No. 5. El factor genético ocupa el primer lugar como causa de amenorrea primaria y representa el $47.7 \%$ (21 pacientes) seguido en su orden por el factor anatómico $29.5 \%$ y el endocrino $22.8 \%$.

Tabla No. 5

\section{DISTRIBUCION DE 44 PACIENTES} CON AMENORREA PRIMARIA

SEGUN LA CAUSA ETIOLOGICA

H.U.S.V. DE P. - U. DE A., 1976-1979

\begin{tabular}{rlcr}
\hline & & \multicolumn{2}{c}{$\begin{array}{c}\text { Número Total } \\
\text { de }\end{array}$} \\
pacientes
\end{tabular}

\section{DISTRIBUCION DE 44 PACIENTES CON AMENORREA PRIMARIA SEGUN LA CAUSA ETIOLOGICA H.U.S.V. - U. DE. A 1976-1979

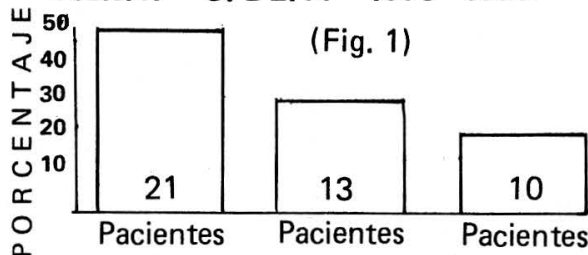 GENETICA \\ ENDOCRINA}

\section{ANATOMICA}

En la Tabla No. 6 las pacientes se clasifican de acuerdo al diagnóstico etiológico. Puede observarse que la causa genética más frecuente es la disgenesia gonadal con un $90.5 \% \quad$ (21 pacientes).

El síndrome de Mayer-RokitanskyKüster-Hauser (MRKH) representa el $69.2 \%$ de la causa anatómica. Los síndromes de anovulación crónica: ovario poliquístico e hiperplasia suprarrenal congénita ocupan el $80 \%$ de la causa endocrina.

La Tabla No. 7 muestra el orden de frecuencia de las causas etiológicas de la 
amenorrea primaria. Le corresponde el primer lugar a la disgenesia gonadal $43.2 \%$ y le siguen el síndrome de

Fig. 2

\section{DISTRIBUCION DE 44 PACIENTES CON AMENORREA PRIMARIA SEGUN CAUSA ETIOLOGICA Y DIAGNOSTICO CLINICO HUSVP-U. DE. A. 1976-1979}

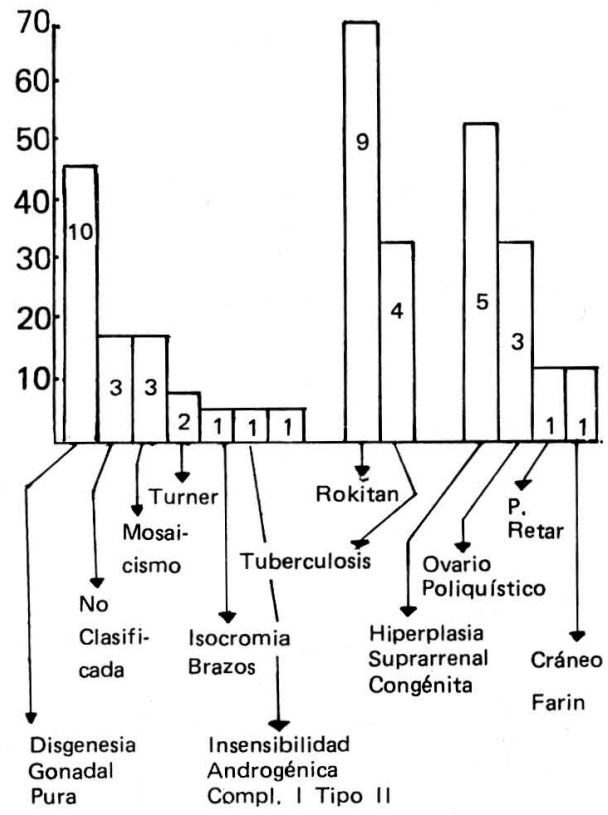

\section{GENETICA ANATOMICA ENDOCRINA}

MRKH. $(20.5 \%)$, la hiperplasia suprarrenal congénita. $(11.4 \%)$, la tuberculosis genital $(9.0 \%)$ y el síndrome de ovario poliquístico $(6.8 \%)$. Con menor frecuencia el craneofaringioma y la pubertad retardada, con un $2.3 \%$.

Al analizar la causa genética, Tabla No. 8, la disgenesia gonadal pura $X X$ ocupa el primer lugar $(61.9 \%)$. Siguen en orden de frecuencia el mosaicismo y el síndrome de Turner. Sólo a una paciente se le diagnosticó isocromia de
Tabla No. 6

DISTRIBUCION DE 44 PACIENTES CON AMENORREA PRIMARIA SEGUN EL DIAGNOSTICO CLINICO H.U.S.V. DE P. - U. DE A., 1976-1979

\begin{tabular}{lcc}
\hline Causa Etiológica & $\begin{array}{l}\text { Número de } \\
\text { Pacientes }\end{array}$ & Porcentaje \\
\hline CONGENITA & 19 & $(90.5 \%)$ \\
Disgenesia Gonadal & 2 & \\
$\begin{array}{l}\text { Síndrome de Insensibilidad } \\
\text { Androgénica }\end{array}$ & & \\
ANATOMICA & 9 & $(69.2 \%)$ \\
Síndrome de Rokitansky & 9 & \\
Tuberculosis Genital & 4 & \\
$\begin{array}{l}\text { ENDOCRINA } \\
\text { Hiperplasia Suprarrenal } \\
\text { Congénita }\end{array}$ & 5 & $(50.0 \%)$ \\
S. de Ovario Poliquístico & 3 & \\
Pubertad Retardada & 1 & \\
Craneofaringioma & 1 & \\
\hline \multicolumn{1}{c}{ TOTAL: } & 44 & \\
\hline
\end{tabular}

brazos largos. Tres pacientes con diagnóstico de disgenesia gonadal pura $(14.3 \%)$ no fueron clasificadas, debido a que no se logró estudiar el cariotipo, una de ellas presentaba cromatina negativa.

En el grupo de pacientes con insensibilidad androgénica, una paciente se clasificó como un síndrome de feminización testicular y otra como un síndrome incompleto tipo II (hipospadia perineoescrotal seudo vaginal) a quien se le diagnosticó un seminoma por estudio anatomo-patológico.

La Tabla No. 9 muestra la talla promedio de las pacientes con disgenesia gonadal: se aprecia que en la disgenesia gonadal pura se alcanza una talla mayor (1.58 $\mathrm{mts}$ en promedio) mientras que en las pacientes con alteraciones del cromosoma $X$, la talla es de $1.45 \mathrm{mts}$ ó menos. 
Tabla No. 7

\section{CLASIFICACION DE 44 PACIENTES CON AMENORREA PRIMARIA \\ SEGUN LAS DIFERENTES \\ ENTIDADES CLINICAS EN \\ ORDEN DE FRECUENCIA}

H.U.S.V. DE P. - U. DE A., 1976-1979

\begin{tabular}{lc}
\hline & $\begin{array}{l}\text { Número de } \\
\text { pacientes }\end{array}$ \\
\hline Disgenesia Gonadal & $19(43.2 \%)$ \\
Síndrome de Rokitansky & $9(20.5 \%)$ \\
Hiperplasia Suprarrenal & $5(11.4 \%)$ \\
Tuberculosis Genital & $4(9.1 \%)$ \\
$\begin{array}{l}\text { Síndrome de Ovario } \\
\text { Poliquístico }\end{array}$ & $3(6.8 \%)$ \\
$\begin{array}{l}\text { Síndrome de insensibilidad } \\
\text { Androgénica }\end{array}$ & $2(4.7 \%)$ \\
Pubertad Retardada & $1(2.3 \%)$ \\
Craneofaringioma & $1(2.3 \%)$ \\
\hline \hline
\end{tabular}

Figura No. 3

CLASIFICACION DE 44 PACIENTES CON AMENORREA PRIMARIA DE ACUERDO A LA ENTIDAD CLINICA HUSVP - U. de A. 1976-1979

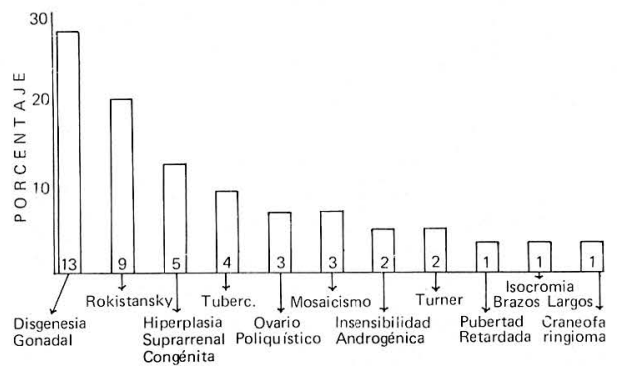

Tabla No. 8

CLASIFICACION DE 19 PACIENTES CON AMENORREA PRIMARIA SEGUN LA CAUSA GENETICA -

H.U.S.V. DE P. - U. DE A., 1976-1979

Disgenesia Gonadal -

Pura $X X$

$10(47.6 \%)$

Disgenesia Gonadal no

clasificada

$3(14.3 \%)$

Mosaicismo $(\mathrm{XX} / \mathrm{X})$

$3(14.3 \%)$

Turner

$2(9.5 \%)$

Isocromía de Brazos largos $1(4.8 \%)$

Insensibilidad Androgénica completa

$1(4.8 \%)$

Incompleta Tipo II

$1(4.8 \%)$

TOTAL:
Tabla No. 9

\section{CLASIFICACION DE 19 PACIENTES} CON DISGENESIA GONADAL DE ACUERDO A LA TALLA H.U.S.V. DE P. - U. DE A., 1976-1979

\begin{tabular}{lrc}
\hline & $\begin{array}{c}\text { Número } \\
\text { pacientes }\end{array}$ & $\begin{array}{c}\text { Talla Promedio } \\
\mathrm{cm}\end{array}$ \\
\hline $\begin{array}{l}\text { Pura } \\
\text { Isocrom ía de Brazos }\end{array}$ & 13 & 153.8 \\
Largos & 1 & 145 \\
Mosaico & 3 & 144 \\
Turner & 2 & 134 \\
\hline \hline
\end{tabular}

Al analizar la causa anatómica, Tabla No. 6, la mayor frecuencia corresponde a la causa congénita, síndrome de $\mathrm{MRKH}$ con el $69.2 \%$ (9 pacientes) y el $30.8 \%$ 
Tabla No. 10

\begin{tabular}{|c|c|c|c|c|c|c|c|c|}
\hline \multicolumn{3}{|c|}{ CLASIFICACIO } & \multicolumn{4}{|c|}{$\begin{array}{l}\text { DE LAS PRINCIPALES CARACTERISTIC } \\
\text { CON EL SINDROME DE ROKITANSKY } \\
\text { H.U.S.V. DE P. - U. DE A., - 1976-1979 }\end{array}$} & \multirow[b]{2}{*}{$\begin{array}{l}\text { Cariotipo } \\
\text { Cromatina }\end{array}$} & \multirow[b]{2}{*}{$\begin{array}{l}\text { Neovagina } \\
\text { Quirúrgica }\end{array}$} \\
\hline Nombre & Edad & Talla & $\begin{array}{l}\text { Mamas } \\
\text { Tanner }\end{array}$ & Genitales & Malformaciones & $\begin{array}{l}\text { Urografía } \\
\text { Secretora }\end{array}$ & & \\
\hline ML & 20 & 142 & V & $\begin{array}{l}\text { Saculación } \\
\text { Vaginal }\end{array}$ & No & No & $46 \times X / 11 \%$ & No \\
\hline MJ & 22 & 152 & V & $\begin{array}{l}\text { Agenesia } \\
\text { Vaginal }\end{array}$ & No & No & $20 \%$ & No \\
\hline LA & 18 & 145 & IV & $\begin{array}{l}\text { Agenesia } \\
\text { Vaginal }\end{array}$ & $\begin{array}{l}\text { Vértebra Dorso } \\
\text { Lumbar }\end{array}$ & $\begin{array}{l}\text { Ectopia } \\
\text { Renal } \\
\text { Cruzada }\end{array}$ & $29 \%$ & No \\
\hline$B Z$ & 22 & 150 & IV & $2 \mathrm{~cm}$ Vagina & No & Normal & $20 \%$ & No \\
\hline ES & 31 & -- & V & $2 \mathrm{~cm}$ Vagina & No & Normal & $20 \%$ & $\mathrm{Si}$ \\
\hline RG & 19 & 158 & IV & $2 \mathrm{~cm}$ Vagina & No & Normal & $6 \%$ & No \\
\hline $\mathrm{RH}$ & 18 & $\cdots$ & V & $\begin{array}{l}\text { Agenesia } \\
\text { Vaginal }\end{array}$ & No & Normal & & No \\
\hline LS & 22 & 165 & IV & $\begin{array}{l}\text { Agenesia } \\
\text { Vaginal }\end{array}$ & $\begin{array}{l}\text { Klippel-Feil } \\
\text { Vértebra Cervi- } \\
\text { cal }\end{array}$ & $\begin{array}{l}\text { Ectopia } \\
\text { Renal } \\
\text { Cruzada }\end{array}$ & $46, X X-45, X / 9 \%$ & $\mathrm{Si}$ \\
\hline LP & 20 & 152 & V & $\begin{array}{l}\text { Agenesia } \\
\text { Vaginal }\end{array}$ & $\begin{array}{l}\text { Cardiopatía } \\
\text { (CIA) }\end{array}$ & Normal & & $\mathrm{Si}$ \\
\hline
\end{tabular}

(4 pacientes) a la tuberculosis genital como única causa adquirida.

La Tabla No. 10 resume los hallazgos obtenidos de las pacientes con el síndrome de MRKH quienes tienen una talla promedio de 1.52 metros. En dos pacientes de $9(22 \%)$ se encontraron malformaciones renales (ectopia renal cruzada) asociadas a malformaciones vertebrales. Una de estas pacientes se clasificó como síndrome de Rokitansky-klippel Feil y presentaba un mosaicismo $(46 \times x / 45 x)$; en otra de las pacientes se sospechó mosaicismo al presentar una cromatina sexual del $6 \%$ pero no se confirmó porqué no se practicó el cariotipo. Tres pacientes se intervinieron quirúrgicamente con el fín de practicar neovagina y 4 presentaban saculación vaginal al momento del examen.
Al analizar la causa endocrina (Tabla No. 11) la alteración funcional es la etiología más frecuente así: la hiperplasia suprarrenal congénita ocupa el primer lugar $(50 \%)$, siguiendo en su orden el síndrome de ovario poliquístico $(30 \%)$ y un caso de pubertad retardada. Un sólo caso fue diagnosticado como craneofaringioma y fue fatal durante la intervención quirúrgica.

\section{Discusión}

Aunque son varios los esquemas propuestos para el estudio de la amenorrea primaria, para el clínico es un dilema elegir el más adecuado, pues el interés es el de llegar a un diagnóstico rápido con el mínimo número de exámenes, evitando gastos innecesarios y la prescripción 
Tabla No. 11

\section{CLASIFIEACION DE 10 PACIENTES CON AMENORREA PRIMARIA SEGUN CAUSA ENDOCRINA H.U.S.V. DE P. - U. DE A., 1976-1979}

I. DISFUNCIONAL

$\begin{array}{ll}\text { Hiperplasia Suprarrenal } & 5(50 \%) \\ \text { Síndrome de Ovario } & \\ \text { Poliquístico } & 3(30 \%) \\ \text { Pubertad Retardada } & 1(10 \%)\end{array}$

II. TUMORAL

Craneofaringioma

$1(10 \%)$

empírica de agentes hormonales como tratamiento sintomático.

El plan de estudio de las pacientes con amenorrea primaria, en el cual predomina el análisis clínico, permite la orientación diagnóstica basada en los hallazgos encontrados durante el examen. Rápidamente se llega a un diagnóstico presuntivo y se establecen puntos de etiología y tratamiento. En nuestro estudio, al utilizar este plan, se confirmó el diagnóstico clínico inicial en el $80 \%$ de las pacientes y se facilitó además el diagnóstico final en el $20 \%$ restante.

En el presente estudio se observó que sólo un número muy bajo de pacientes con amenorrea primaria llegan a la consulta ginecológica especializada $(4 \%)$; sin embargo no por esto dicha alteración carece de importancia clínica. La falta de un criterio definido para el diagnóstico y tratamiento de estas pacientes hace que tengan un promedio mayor de consultas, que pacientes con otras entidades clínicas. A veces por dilación en tomar una conducta adecuada se corre el peligro de que progresen lesiones expansivas intra y extra selares; así mismo, la disgenesia gonadal $X Y$ puede evolucionar a procesos malignos. Esta misma dilación hace que no se preste una adecuada $y$ oportuna asistencia sicológica a pacientes con infantilismo o indiferenciación sexual.

En el presente estudio también se observó que la frecuencia de las causas etiológicas de la amenorrea primaria está de acuerdo con los diferentes informes presentados por otros investigadores (8), resultado obtenido por una metodología diferente.

Las pacientes las hemos clasificado según causa genética, anatómica y endocrina, por considerar que esta clasificación es muy didáctica, a diferencia de otras que dividen en central ovárica, uterina y vulvar.

La disgenesia gonadal como consecuencia de alteración crosómica (mosaicismo, isocromia del brazo largo del cromosoma $X$, o la ausencia de una cromosoma $\mathrm{X}$ ) está asociada a baja estatura, hallazgo que tiene como punto común la falta del brazo corto del cromosoma $X$, donde posiblemente se encuentra localizado alguno de losgenes determinante de la talla (9). Este hallazgo observado en el presente trabajo en donde el síndrome de Turner alcanzó un promedio de 1.34 metros mientras que en el mosaicismo, el promedio más alto es de 1.44 metros, siempre inferior a la talla alcanzada por la disgenesia gonadal pura.

En el presente estudio las 13 pacientes con disgenesia gonadal pura tenían un promedio de talla de 1.53 , el cual es inferior al informado por otros autores. Este hallazgo puede explicarse por el hecho de que en nuestra población los percentiles de talla están por debajo de lo informado en países más desarrollados.

A diferencia de las pacientes con síndrome de Turner la disgenesia gonadal pura tiende a manifestarse en más de un miembro de la familia (10), pues su transmisión se hace en forma autosómica 
recesiva o recesiva unida al cromosoma $X(10-11)$. En este informe sólo a 2 hermanas se les diagnosticó disgenesia gonadal pura $X X$.

Según algunos informes de la literatura la mayor frecuencia de la disgenesia gonadal pura tiene un complemento cromosómico $X X$ y sólo el $10 \%$ es $X Y$ (Síndrome de Swyer). En el presente estudio no se encontró ningún caso $X Y$.

La disgenesia gonadal pura $X Y Y$ aquellas entidades con fenotipo femenino $y$ con complemento cromosoma $Y$, tales como el síndrome de insensibilidad androgénica, disgenesia gonadal mixta y hermafroditismo verdadero, presentan un índice muy alto de malignización de la gonada, que es mayor y más precoz, en su aparición cuanto más indiferenciada sea ésta (12).

A dos pacientes de este estudio se les diagnosticó un síndrome de insensibilidad androgénica. Una de ellas era un testículo feminizante completo y la otra un testículo feminizante tipo II (hipospadia perineo escrotal seudo vaginal), a esta última se le diagnosticó un seminoma a la edad de 25 años.

Los resultados obtenidos en el presente estudio y los informados en la literatura están de acuerdo con la menor frecuencia del síndrome de Turner, $(10.5 \%)$. Sin embargo, este síndrome, por los hallazgos clínicos tan evidentes, permanece como el modelo clásico para referirse a la disgenesia gonadal.

Es importante destacar que no sólo las alteraciones de los cromosomas sexuales son responsables de la disgenesia gonadal, sino también las alteraciones (delecciones) a nivel de los autosomas 13 y 18 (13).

En el presente estudio las malformaciones anatómicas ocuparon el segundo lugar como causa de amenorrea primaria y le correspondió al síndrome de Mayer Rokitansky Kuster Hauser el primer lugar en este grupo $(69.2 \%)$. Es interesante observar que esta última entidad ocupa el segundo lugar como causa de amenorrea primaria, al discriminar la disgenesia gonadal en sus diferentes entidades cl ínicas (Tabla No. 12). En el presente estudio las pacientes con el síndrome de MRKH consultaron tardíamente, promedio de 21 años de edad; esta actitud es el resultado de la presencia en la paciente de caracteres sexuales secundarios, a diferencia de aquellas con infantilismo, indiferenciación sexual o alteración en los conductos del seno urogenital, que consultan más precozmente.

Tabla No. 12

\section{CLASIFICACION DE 44 PACIENTES CON AMENORREA PRIMARIA \\ SEGUN LA ENTIDAD CLINICA ESPECIFICA EN ORDEN DE FRECUENCIA}

H.U.S.V. DE P. - U. DE A., 1976-1979

\begin{tabular}{lll}
\hline Disgenesia Gonadal Pura & 13 & $(29.5)$ \\
$\begin{array}{l}\text { Mayer-Rokitansky-Kuster- } \\
\text { Hauser }\end{array}$ & 9 & $(20.4)$ \\
$\begin{array}{l}\text { Hiperplasia Suprarrenal } \\
\text { Congénita }\end{array}$ & 5 & $(11.4)$ \\
$\begin{array}{l}\text { Tuberculosis Genital } \\
\text { Síndrome de Ovario }\end{array}$ & 4 & $(9.1)$ \\
$\begin{array}{l}\text { Poliquístico } \\
\text { Mosaicismo }\end{array}$ & 3 & $(6.8)$ \\
Insensibilidad Androgénica & 2 & $(4.5)$ \\
Síndrome de Turner & 2 & $(4.5)$ \\
Pubertad Retardada & 1 & $(2.3)$ \\
Isocromía de Brazos & 3 & $(6.8)$ \\
Largos & 1 & $(2.3)$ \\
Craneofaringioma & 1 & $(2.3)$ \\
\hline \hline
\end{tabular}


Tabla No. 13

\title{
CLASIFICACION DE 44 PACIENTES CON AMENORREA PRIMARIA SEGUN EL ORIGEN GONADAL O EXTRAGONADAL
}

\author{
H.U.S.V. DE P. - U. DE A., 1976-1979
}

Disgenesia Gonadal Pura

Disgenesia Gonadal en Mosaico

Síndrome de Turner

Isocromía de Brazos Largos
13

$\begin{array}{ll}\text { Síndrome de Rokitansky } & 9(56.8 \%) \\ \text { Hiperplasia Suprarrenal } & 5\end{array}$

Tuberculosis Genital

2
Síndrome de Ovario Poliqu ístico

Pubertad Retardada

Craneofaringioma

Insensibilidad Androgénica

\section{4 \\ $25(56.8 \%)$ \\ $208(40.4 \%)$}

\section{TOTAL:}

Ross GT (11 autores)

(Williams Text Book)
$19(43.2 \%)$

$310(59.8 \%)$
Por lo anterior se puede concluír que el hallazgo de una alteración uterovaginal con presencia de caracteres sexuales secundarios, en una etapa tardía de la pubertad, determina el diagnóstico de un síndrome de $\mathrm{MRKH}$. De las 9 pacientes con el síndrome de MRKH 2 de ellas presentan simultáneamente malformaciones urológicas y vertebrales; en relación con las alteraciones vertebrales una de las pacientes se clasificó como un síndrome de Rokitansky-Klippel Feil, en donde la agenesia útero-vaginal se asocia con cuello corto, fusión de vértebras cervicales, limitación no dolorosa de los movimientos de la cabeza e implantación baja del cabello (13-16).

Según informes de la literatura las alteraciones cromosómicas en el síndrome de MRKH son raras (14); sin embargo, en el presente estudio se detectó una paciente con cariotipo $46, \times X / 45 X$, con $9 \%$ de cromatina sexual; posiblemente otra paciente con una cromatina sexual del $6 \%$ pudo tener un patrón similar. En estas últimas pacientes el hallazgo de un mosaico, y ante la presencia de ovarios funcionantes, nos hace pesnar que la línea cromosómica predominante es de $46 \mathrm{XX}$, hallazgo cromosómico que suele asociarse a menopausia prematura, posiblemente debido a un número limitado de folículos funcionantes, determinado por la constitución cromosómica (15).

Teniendo en cuenta lo anterior sugerimos que en las pacientes con el diagnóstico de MRKH es aconsejable practicar cariotipo con el fín de investigar alteraciones cromosómicas y establecer así un diagnóstico diferencial con los síndromes incompletos de insensibilidad androgénica. En nuestra opinión el diagnóstico del síndrome de MRKH es el resultado de un análisis clínico adecuado, complementado con el estudio cromosómico.

La tuberculosis genital (TBG) aunque presente en nuestra población como 
consecuencia de las pésimas condiciones existentes, raras veces se piensa en ella como causa de amenorrea primaria, pues su manifestación es muy tórpida; sin embargo, se destaca en este estudio por ser la única causa adquirida de amenorrea.

La tuberculosis genital es la única causa adquirida de amenorrea primaria (16) y es secundaria a un proceso pulmonar o peritoneal; su diagnóstico se hace mediante el empleo de uno o varios métodos, tales como la biopsia de endometrio, histerosalpingografía, laparoscopia o laparatomía; además el cultivo de endometrio o de líquido peritoneal, los cuales no fue posible efectuar en nuestro medio.

En las pacientes con amenorrea primaria al efectuar la biopsia de endometrio, se encontró una atrofia endometrial, que es el resultado de su destrucción por el bacilo tuberculoso. Esta atrofia endometrial se asocia a un adecuado influjo hormonal a nivel de efectores tales como picnosis elevada, moco filante $y$ con helecho, curva de temperatura bifásica.

En estos casos de atrofia endometrial es muy difícil encontrar el bacilo de Koch, pues la atrofia es una lesión residual, secundaria a la lesión tubárica, dando como resultado un mal pronóstico para la fertilidad.

El factor endocrino ocupa el último lugar como causa de amenorrea primaria con el $22 \%$, siendo la hiperplasia suprarrenal la entidad más frecuente; esta entidad tiene diferentes manifestaciones clínicas como consecuencia del grado de déficit enzimático, presentándose en la niñez con diferentes grados de ambiguedad sexual cuando el déficit es severo o durante la pubertad o en la edad adulta, cuando el déficit es parcial (17).

Esta entidad se acompaña de anovulación crónica asociada a trastornos del ciclo, hirsutismo y acné.
De las 5 pacientes con hiperplasia suprarrenal 2 tenían un déficit enzimático severo $y$ por lo tanto presentaban diversos grados de indiferenciación sexual, por lo cual se les practicó cirugía reconstructiva; 3 sólo presentaban amenorrea sin alteraciones anatómicas.

El síndrome de ovario poliquístico como alteración funcional del eje hipotálamo-hipófisis ovario es de carácter progresivo y por lo tanto raras veces es causa de amenorrea primaria; (18) ocupó el segundo lugar en la causa endocrina con una frecuencia del $6.8 \%$ en relación al total de pacientes estudiadas; no se encontraron otras causas de anovulación tales como obesidad, hiperprolactinemia o hipertiroidismo.

Al desglosar las causas de la amenorrea primaria en los diferentes diagnósticos, continúa la causa genética siendo la más frecuente, con la disgenesia gonadal pura, 29.5\%; el síndrome de MRKH con el $20.4 \%$ como causa anatómica y la hiperplasia suprarrenal con el $11.4 \%$, como causa endocrina.

$\mathrm{Si}$ se dividen las pacientes con amenorrea primaria de acuerdo a la causa gonadal o extragonadal, la mayor frecuencia corresponde a este último grupo con un $56.8 \%$, diferente a los datos informados por ROSS (8), debido a que en nuestro estudio el síndrome de ovario políquistico lo clasificamos como de causa extragonadal. Si unificáramos estos criterios los resultados serían sensiblemente iguales.

Es necesario enfatizar la importancia de no administrar ningún tratamiento hormonal antes de confirmar el diagnóstico, pues se corre el riesgo de enmascarar el cuadro clínico. La actitud del médico en estos casos es de dar un estímulo a la paciente para continuar el estudio, iniciar una terapia de apoyo antes de llegar a un diagnóstico definitivo. Se tiene en cuenta que raras veces la entidad 
clínica causal exige un tratamiento de urgencia. La amenorrea no es un diagnóstico sino síntoma de un proceso más complejo que amerita ser diagnóstica-

do.

La importancia del presente estudio fue no sólo el llegar a un diagnóstico etiológico de la amenorrea primaria antes de iniciar el tratamiento médico, sino el predecir su futuro.

1. Se encontraron alteraciones congénitas cardiovasculares en dos pacientes (Turner y MRKH) y vertebrales y renales en otros dos MRKH.

2. La malignización gonadal, se diagnosticó en una paciente $(2.3 \%)$.

3. De acuerdo a la talla, 6 pacientes $(13.6 \%)$, son de baja estatura de causa cromosómica, y el resto, 38 pacientes $86.4 \%$ de estatura normal o elevada.

4. En relación con el pronóstico sexual y obstétrico, 11 pacientes $(25 \%)$, tenían imposibilidad para efectuar un coito (9 pacientes con el diagnóstico del síndrome de Rokitansky, una con insensibilidad androgenica tipo II y una con hiperplasia suprarrenal, las dos últimas con genitales ambiguos).

Un total de 35 pacientes $(79.5 \%)$, son de mal pronóstico obstétrico; sólo 9 pacientes $(20.5 \%)$ son de mejor pronóstico y pertenecen al grupo de causa endocrina.

5. Terapia hormonal: se inició sólo al confirmar el diagnóstico o al menos con las determinaciones hormonales básicas y la toma de algunos exámenes complementarios (edad ósea, cromatina sexual, cariotipo y determinaciones hormonales).

El tratamiento hormonal tiene los siguientes objetivos: a) Desarrollar los caracteres sexuales secundarios, utilizando estradiol a la dosis de $1 \mathrm{mg} / \mathrm{d}$ ía, indefinidamente, hasta obtener un desarrollo adecuado de la glándula mamaria hasta Tanner III, permitiendo al mismo tiempo el desarrollo normal de la talla al acelerar el desarrollo y la disfusión a nivel de la metáfisis, en forma progresiva; posteriormente se inicia una terapia secuencial para desencadenar la menstruación.

b) En aquellas pacientes que tengan una disfunción hipotálamo hipofisiaria, secundaria a una alteración suprarrenal, se prescribe terapia a base de dexametasona, 0.5 a $0.75 \mathrm{mg}$ a las 11 p.m. por 15 a 30 días hasta obtener una excresión de 17 cetosteroides en orina de 24 horas por debajo de $10 \mathrm{mg}$; con esta terapia, cuando se inicia antes de los 20 años, se obtiene desarrollo de los caracteres sexuales, menstruaciones y ocasionalmente ovulación o ciclos anovulatorios, cuando la terapia es más tardía.

En los casos en que se diagnostica un síndrome de anovulación crónica con estro persistente, se da una terapia, cíclica cada mes a base de progestágenos (10 mg del 21 al 25 ciclo), con el fin de desencadenar menstruaciones.

En los casos de atrofia endometrial se debe descartar fuera de la hiperplasia suprarrenal, los estados de hiperprolactinemia, $y$ en este caso el tratamiento es a base de drogas dopaminérgicas.

c) En raras ocasiones se logra la ovulación en pacientes con amenorrea primaria (sólo en las de causa endocrina).

d) Algunas de estas pacientes necesitan evaluación siquiátrica; la mayoría sólo necesitó el apoyo sicológico del médico tratante, orientado a explicarles su tratamiento y el futuro obstétrico y ginecológico. 


\section{STUDY OF PRIMARY AMENORRHEA DURING A FOUR YEARS PERIOD}

\section{Summary:}

53 patients with primary amenorrhea were analyzed during a four years period, from 1976 to 1979 , applying a deductive clinical method which we think is very usefull in practice. The frequency of primary amenorrhea is $4 \%$ in a reference service; 44 of the 53 patients who looked for consultation were studied and the results showed the highest frequency in the genetic group, followed, in their order, by the anatomic and endocrine causes. Pure gonad dysgenesis syndrome was the predominant clinical entity, followed by the Rokytansky syndrome.

Tuberculosis, as unique entity obtained, shows a $9.1 \%$ frequency. The patients with the best prognosis regarding fertility correspond to the group of endocrine cause.

\section{Bibliografía}

1. ZARATE A. (Información personal).

2. SANCHEZ F., GARCIA J. Manejo clínico de la amenorrea primaria (en prensa).

3. SANCHEZ F., GARCIA J. Alteraciones del ciclo menstrual: Amenorrea en Ginecología y Obstetricia, texto integrado 2a. ed. pág. 3 1980, Ed. U. de A. Medellín.

4. KUPPERMAN Hs, LEFKOVICS (1957) Progesterone in problem of sterility; diagnostic and therapeutic use: Fertil Steril 8: 131.

5. GILSON MD, KNAB DR (1973) primary amenorrhea. A simplified approach to diagnosis, Am. J. Obstet 117-400.

\section{Resumen}

Se analizan 53 pacientes con amenorrea primaria durante un período de 4 años, 1976-1979, utilizando un método clínico deductivo, el cual creemos es de mayor utilidad práctica. La frecuencia de amenorrea primaria es de un $4 \%$ en un servicio de referencia. De 53 pacientes que consultaron 44 fueron estudiadas, correspondiendo la mayor frecuencia al grupo genético, siguiéndole en su orden la causa anatómica y la endocrina. El síndrome de disgenesia gonadal pura fue la entidad clínica predominante, seguido del síndrome de Rokitansky.

La tuberculosis como única entidad adquirida presenta una frecuencia alta de $9.1 \%$. Las pacientes con mejor pro nóstico en relación a la fertilidad pertenecen al grupo de causa endocrina.

6. ZARATE A., CANALES ES (1972). Estudio de la paciente con amenorrea. Rev. Medic1MSS 11: 201.

7. NAKANO R. HASHIBA N. KOTSUJIF. TOJOS: (1977) A. squematic approach to the work-up of amenorrea, Fertil Steril 28: 229.

9. Mc. DONOUGH P. (1972), Disgenesia Gonadal y sus variantes. Clin. Nort. Am. 19:631.

8. ROSS, G.T. y VANDE WIELE, R.L. The ovaries en Texbook of endocrinology, 5a. ed. Williams, R. M. Ed. Filadelfia, WB Saunders, 1974.

10. SIMPSON JL, CHRISTAKOS A. HORWIT M, SILVERMAN FS. Gonadal.dysgenesis in individuals with aparently nor- 
mal chromosomal complements: Tabulation of cases and compilation of genetic date in Birth, vol. VII pág. 215.

11. BRUNETEAU DW, SIPAHIOGLU IB, BYRD JR, GREEBBLAT RB (1976), Pure gonadal dysgenesis with and Report of two cases. Am. J. Obstet.: Gynecol 124:255.

12. MANUEL M., KATAYAMA, P., JONES HUNT (1976) Age of ocurrence of gonadal tumors in intersex patients with aY Chromosome. Am. J. Obstet Gynecol 124: 293.

13. RAMSEY J. BLIZNAK J. (1971), KLIP. PEL. Syndrome with renal agenesis and, others anomalies. Am J. Roetgenol Radium ther Nucl Med. 113:460.

14. RODRIGUEZ S., JIMENEZ M., FORSBACH, G., CANALES, SORIA J. ZARATE, A. (1977), Síndrome de MayerRokitansky-Kuster-Hauser de tipo familiar Gacet Medic Mex 113: 447.

15. SCOTT R.B., CAMERON, J.S., OGG, S. (1971). XO/XX mosaicism in the Roki-
tansky-Kuster-Hauser syndrome. Lancet 2: 1380.

16. INDUÑATE, DM, CORREA, CC, ARREOLA, MA, LOPEZ, J.J. HERNANDEZ AYUP S. (1979) amenorrea primaria de etiología fimica. Ginec. Obstet. México 46: 363.

17. JUBIZ W. The Adrenals en Endocrinology 1a. ed., Mc Graw Hill Ed. New York, pág. 801979.

18. CANALES, E.S., ZARATE, A., CASTELAZO-AYALA L. 1971. Primary amenorrhea associated with polycystic ovaries: endocrine, cytogenetic and thera peutic considerations Obstet, Gynec. 37: 205.

19. DEL CORRAL F., ZUÑIGA, J.E., (1975) Amenorrea-ponencia oficial. Rev. Colomb. Ginecol 26:97.

20. CARDONA F., GARCIA J., VASSEUR DE P. O. Amenorrea y la prueba de la progesterona, Pregnandiol, Rev. Col. Obstet. Ginec. 26: 157 